\title{
The effect of geometrical confinement and chirality on domain wall pinning behavior in planar nanowires
}

L. K. Bogart, D. S. Eastwood, and D. Atkinson

Citation: Journal of Applied Physics 104, 033904 (2008);

View online: https://doi.org/10.1063/1.2961313

View Table of Contents: http://aip.scitation.org/toc/jap/104/3

Published by the American Institute of Physics

\section{Articles you may be interested in}

Domain wall pinning and potential landscapes created by constrictions and protrusions in ferromagnetic nanowires

Journal of Applied Physics 103, 114307 (2008); 10.1063/1.2936981

Controlling domain wall pinning in planar nanowires by selecting domain wall type and its application in a memory concept

Applied Physics Letters 92, 022510 (2008); 10.1063/1.2832771

Controlling the stability of both the structure and velocity of domain walls in magnetic nanowires

Applied Physics Letters 109, 062405 (2016); 10.1063/1.4960201

Direct observation of domain-wall pinning at nanoscale constrictions

Applied Physics Letters 87, 102509 (2005); 10.1063/1.2042542

Artificial domain wall nanotraps in $\mathrm{Ni}_{81} \mathrm{Fe}_{19}$ wires

Journal of Applied Physics 95, 6717 (2004); 10.1063/1.1652391

Control of the magnetic vortex chirality in Permalloy nanowires with asymmetric notches

Journal of Applied Physics 116, 193902 (2014); 10.1063/1.4902008

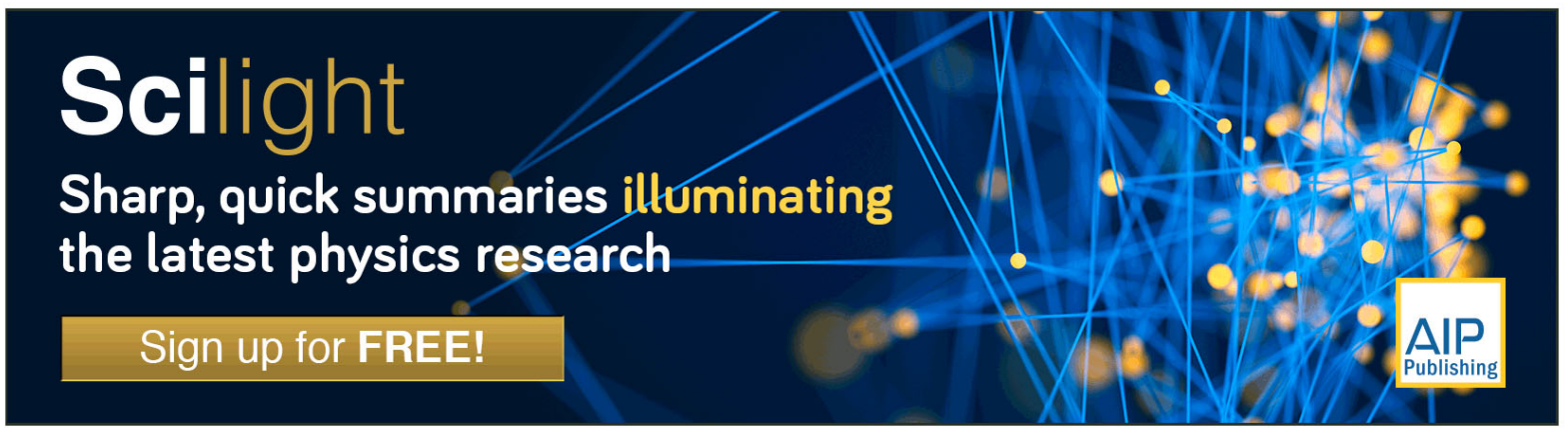




\title{
The effect of geometrical confinement and chirality on domain wall pinning behavior in planar nanowires
}

\author{
L. K. Bogart, ${ }^{\text {a) }}$ D. S. Eastwood, and D. Atkinson \\ Department of Physics, Durham University, Durham DH1 3LE, United Kingdom
}

(Received 7 March 2008; accepted 23 May 2008; published online 1 August 2008)

\begin{abstract}
We investigate the domain wall pinning behavior in Permalloy nanowires using experimental measurements and micromagnetic simulations. Planar nanowire structures were fabricated by electron beam lithography followed by thin-film deposition via thermal evaporation. The magnetization switching behavior of individual nanowires was measured using the magneto-optical Kerr effect. For symmetrical pinning structures such as the junction between a wider domain wall injection pad and a narrower nanowire, the domain wall depinning field increases as the wire width decreases, with the depinning field increasing rapidly for wires widths below $400 \mathrm{~nm}$. For domain wall pinning at asymmetrical structures such as a notch, the magnitude of the depinning field appears relatively insensitive to notch geometry for triangular and rectangular notch structures, compared to the influence of the wire width. The domain wall depinning field from triangular notches increases as notch depth increases although this increase levels off at notch depths greater than approximately $60 \%$ wire width. The nature of domain wall pinning at asymmetrical notch structures is also sensitive to domain wall chirality. () 2008 American Institute of Physics.

[DOI: $10.1063 / 1.2961313$ ]
\end{abstract}

\section{INTRODUCTION}

The behavior of magnetic domain walls in planar nanowires structures has recently attracted much attention with potential applications including both spintronic $\operatorname{logic}^{1}$ and magnetic memory devices. ${ }^{2}$ Such devices require a high level of control of domain wall propagation behavior, which can be achieved through local pinning centers that provide well defined stable wall locations. Artificially structured variations in the nanowire geometry such as notches can be introduced to engineer domain wall pinning and have allowed some of the more fundamental properties of domain walls to be determined. Understanding and controlling the field driven motion of domain walls in nanowires is an important preliminary step to studying their current driven dynamics. ${ }^{3}$ Quantitative correlation between the depinning field and pinning site geometry is highly relevant to both field and spinpolarized current induced domain wall behavior and may ultimately lead to optimizing the control and efficiency of these devices. Understanding the influence of wire width on wall pinning behavior is critical to studies of scaling of planar nanowires for device applications.

\section{EXPERIMENT DETAILS}

Planar magnetic nanowire structures were fabricated using electron beam lithography followed by thin-film deposition and lift-off on a thermally oxidized silicon substrate. Thin films were deposited using thermal evaporation from a single $\mathrm{Ni}_{81} \mathrm{Fe}_{19}$ source, utilizing a base pressure of $10^{-7}$ Torr and a growth pressure of $10^{-6}$ Torr at a rate of $\sim 0.5 \AA / \mathrm{s}$ as measured by an in situ quartz crystal. Actual film thickness was externally verified using $\mathrm{x}$-ray reflectivity measurements

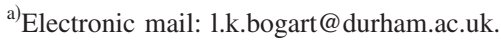

and was within $2 \%$ of the nominal thickness value. Each nanowire structure contained a micron-scale injection pad at one end ${ }^{4}$ to nucleate domain walls and was sharply tapered at the other ${ }^{5}$ to annihilate domain walls; this ensured domain wall propagation in the same direction on successive field reversal cycles. Nominal wire widths ranged from 1000 down to $200 \mathrm{~nm}$ with each structure containing a single asymmetric pinning site patterned along one side of the wire. Pinning behavior was investigated at the symmetric pad-wire junction and the pinning potentials of two different pinning site geometries were investigated in the form of triangular and rectangular shaped notches. Figure 1 shows a scanning electron microscopy (SEM) image of a nanowire structure together with an example of each notch structure.

Spatially resolved magnetization switching measurements were performed using a high sensitivity longitudinal focused magneto-optic Kerr effect (MOKE) magnetometer using an alternating magnetic field at $23 \mathrm{~Hz}$ and utilizing a laser spot focused to an average width of $5 \mu \mathrm{m}$. ${ }^{6}$ The use of a focused laser spot allowed individual nanowire structures to be investigated and the nanowire length was such that the MOKE laser could locally probe the nature of magnetization reversal behavior at both pad-wire junctions and after the notch pinning sites. Further insight into the domain wall pinning behavior was obtained through micromagnetic simula-

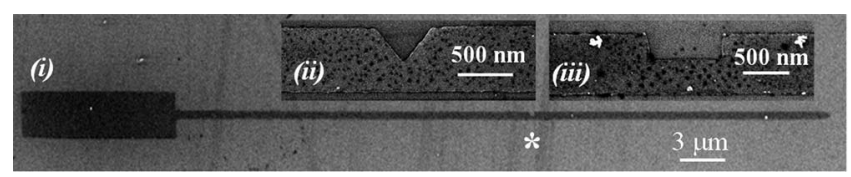

FIG. 1. (i) SEM image of a planar nanowire structure fabricated by electron beam lithography, where ${ }^{*}$ indicates the location of the pinning site. The inset shows examples of a (ii) triangular and (iii) rectangular notch structures. 


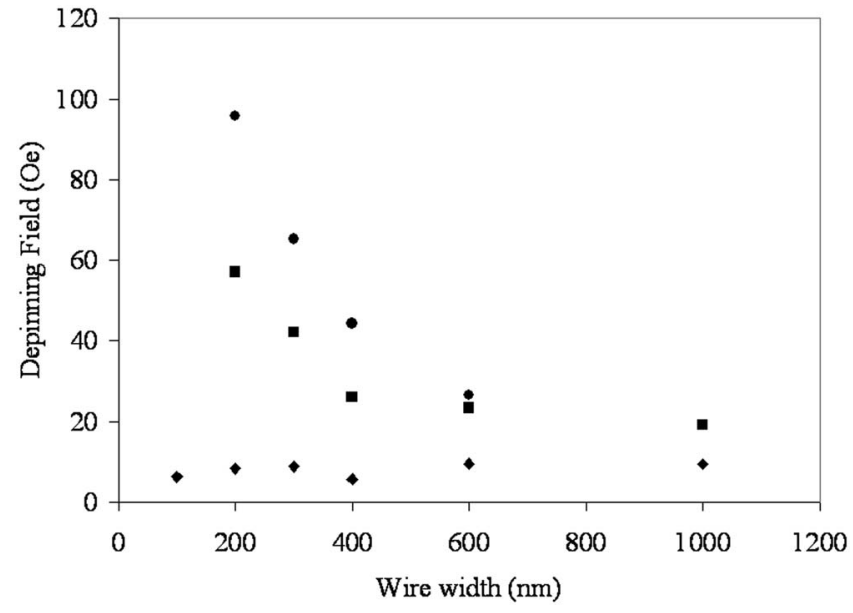

FIG. 2. Switching field as a function of wire width of a $5 \mathrm{~nm}$ thick Permalloy nanowire. ( ) represents magnetization reversal of the nucleation pad, ) represents domain wall injection from the nucleation pad into the wire, and $(\bullet)$ represents domain wall depinning from a triangular notch patterned along one side of the nanowire.

tions, which were performed using the OOMMF micromagnetic software. ${ }^{7}$ All simulations utilized a $5 \mathrm{~nm}$ mesh and employed standard magnetic parameters for Permalloy including saturation magnetization $M_{S}=800 \times 10^{3} \mathrm{~A} / \mathrm{m}$, an exchange energy constant $A=13 \times 10^{-12} \mathrm{~J} / \mathrm{m}$, and zero magnetocrystalline anisotropy.

\section{RESULTS AND DISCUSSION}

A systematic investigation into the relationship between wire width, notch geometry, and field driven domain wall pinning behavior is described here. The magnetic field dependence of domain wall depinning from both injection pad structures and triangular notches as a function of wire width for $5 \mathrm{~nm}$ thick nanowires is presented in Fig. 2. For each injection pad structure, we measure an average switching field of approximately $12 \mathrm{Oe}$, which has no dependence upon nanowire width since this is determined by the magnetostatics of the pad. Once the pad switches, a domain wall is pinned at the pad-wire junction until the applied magnetic field increases; by placing the focused laser spot at the padwire junction, the field needed to depin the wall and "inject" it into the nanowire can be measured. As the wire width decreases the domain wall "injection" field increases, and this increase is much more rapid for wire widths smaller than $400 \mathrm{~nm}$. Once the wall is "injected" from the pad into the wire, it propagates along the length of the nanowire to a notch, the depth of which being equal to approximately half the wire width. For wire widths below $800 \mathrm{~nm}$, the depinning field for the domain wall from the notch is found to be larger than the depinning field from the pad-wire junction, and again the increase in depinning field is much more rapid for wire widths below $400 \mathrm{~nm}$. Similar behavior was observed for $10 \mathrm{~m}$ thick nanowires. Figure 3 shows the domain wall energy per unit cross-sectional area of the nanowire as a function of nanowire width, deduced from micromagnetic simulations of $10 \mathrm{~nm}$ thick nanowires. ${ }^{8}$ As the wire width decreases, we observe that the domain wall energy increases and this increase is much more rapid for wire widths below

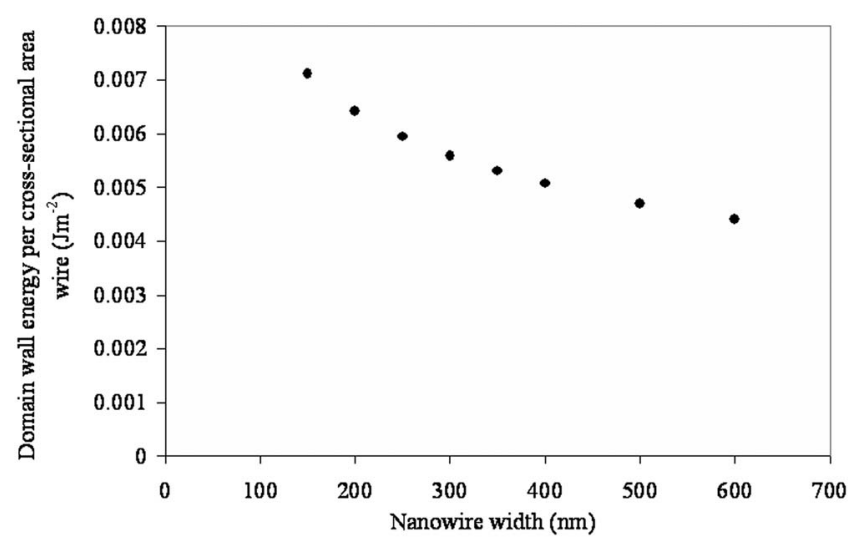

FIG. 3. Plot of domain wall energy per unit cross-sectional area as a function of nanowire width, deduced from OOMMF micromagnetic simulations. ${ }^{8}$

$400 \mathrm{~nm}$. Treating a domain wall pinned at the pad-wire interface as a particle trapped in a potential well, the depth of the pinning potential increases rapidly as the wire width decreases since the energy per unit area required to support a wall increases. It is well known that the micromagnetic structure of a wall that can be supported in a nanowire is highly sensitive to the transverse dimensions of the wire. 9,10 At large wire widths, a vortex wall can be supported; whereas at narrower widths, the wall becomes transverse in nature. Both Figs. 2 and 3 indicate that narrower wire widths require a larger applied magnetic field to inject a wall.

In order to study the effect of notch geometry on domain wall pinning behavior, we have fabricated a set of nanowires containing single notches of both triangular and rectangular shape. The magnetic field required to depin a wall from triangular $(\mathbf{\Delta})$ and rectangular $(\square)$ notches of nominally identical depth as a function of wire width in $10 \mathrm{~nm}$ thick nanowires is presented in Fig. 4. This figure also shows the depinning fields for domain walls injected from the pad into each wire. Domain walls are strongly pinned by both notch structures since such as the pad-wire junction, the notch also represents a potential well from which the domain wall requires energy in the form of an increasing magnetic field to depin. ${ }^{11}$ It is interesting to note that although at first glance

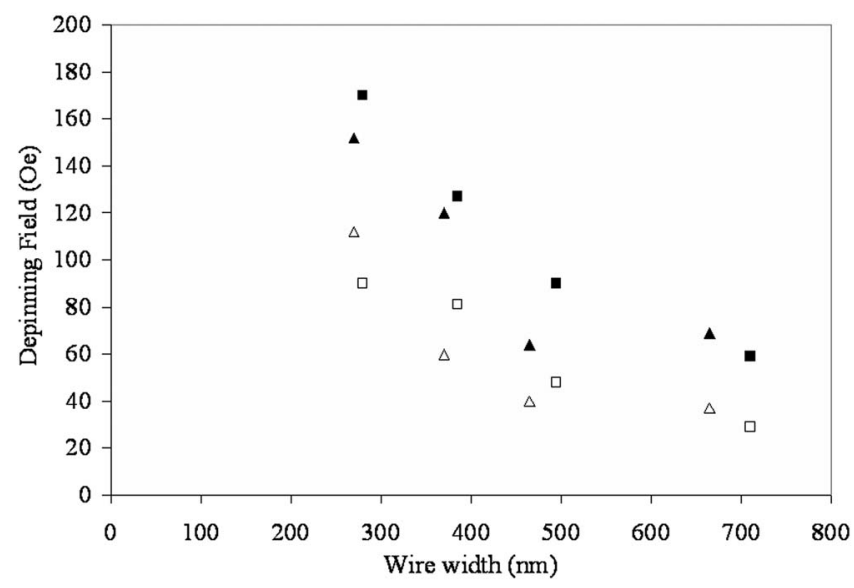

FIG. 4. Injection fields (open symbols) and notch depinning data (solid symbols) for $(\square)$ rectangular notches and $(\boldsymbol{\Lambda})$ triangular notches respectively measured by MOKE. All notches have a nominal depth half that of the wire width. 

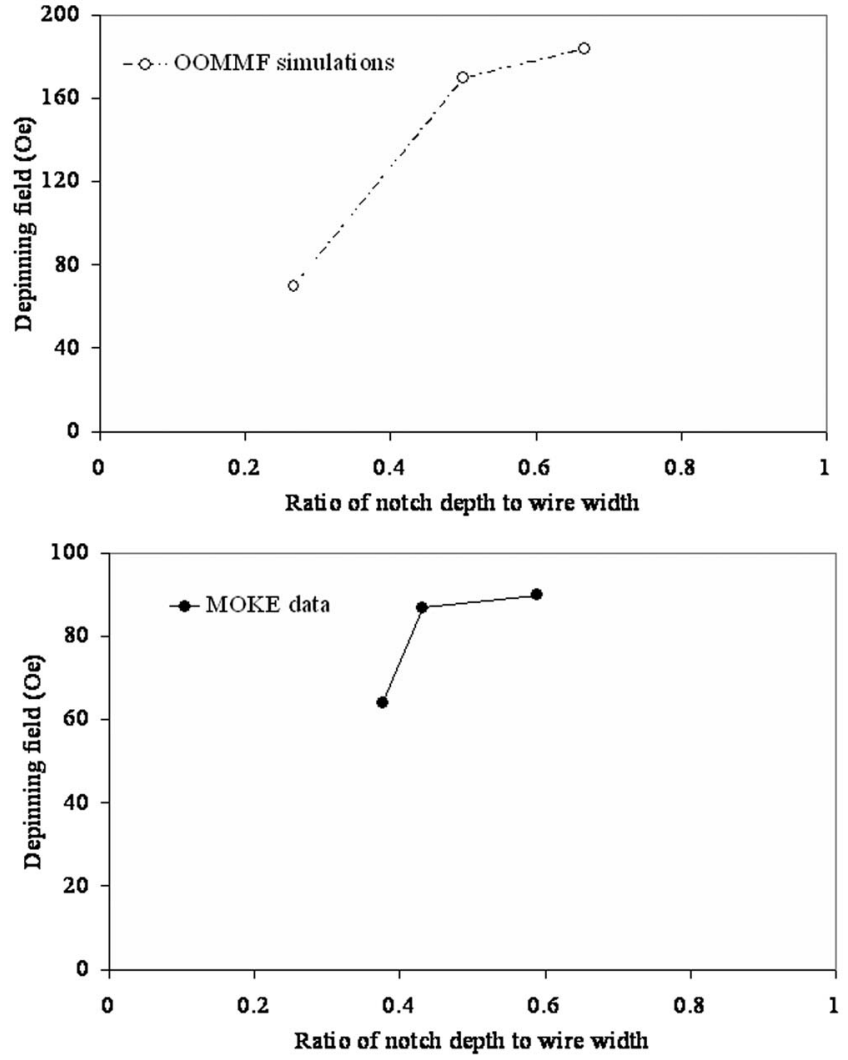

FIG. 5. Domain wall depinning fields as a function of triangular notch depth as deduced from OOMMF micromagnetic simulations for a $300 \mathrm{~nm}$ wide nanowire (top panel) and for a $480 \mathrm{~nm}$ wide nanowire as measured by MOKE hysteresis data (bottom panel).

the rectangular notches pin walls more strongly than their triangular counterparts, all data sets follow the same trend, as seen in Fig. 2, which shows that the wire width is the most important factor. This suggests that the specific geometry for single asymmetric notch structures is of secondary importance and it is the wire width that is the governing factor in determining the depinning field.

The effect of increasing notch depth on the magnitude of domain wall depinning field is presented in Fig. 5. The figure shows the magnitude of depinning fields from triangular notches of increasing depth in a $480 \mathrm{~nm}$ wire as measured by MOKE and micromagnetic simulations of pinning in a 300 $\mathrm{nm}$ wire with notches of increasing depths. The depinning field has a strong dependence on the notch depth. When the notch depth increases from approximately $25 \%$ wire width to $50 \%$ wire width both data sets show an initial increase in depinning field which is in good agreement with Refs. 12 and 13. Both sets of data approach a plateau in the magnitude of depinning field, suggesting a limit to the pinning strength of a single notch structure. For notches deeper than approximately $60 \%$ wire width, we see that further increases in depth do not yield significantly deeper pinning potential. The similar trend in both the experimental data for the 480 $\mathrm{nm}$ wide wire and the simulations for the $300 \mathrm{~nm}$ wide wire suggests that a pinning depth "saturation" effect occurs for a range of wire widths. The pinning site "saturation pinning field" is determined by the wire width and these results suggest that the pinning site saturation will occur at a similar

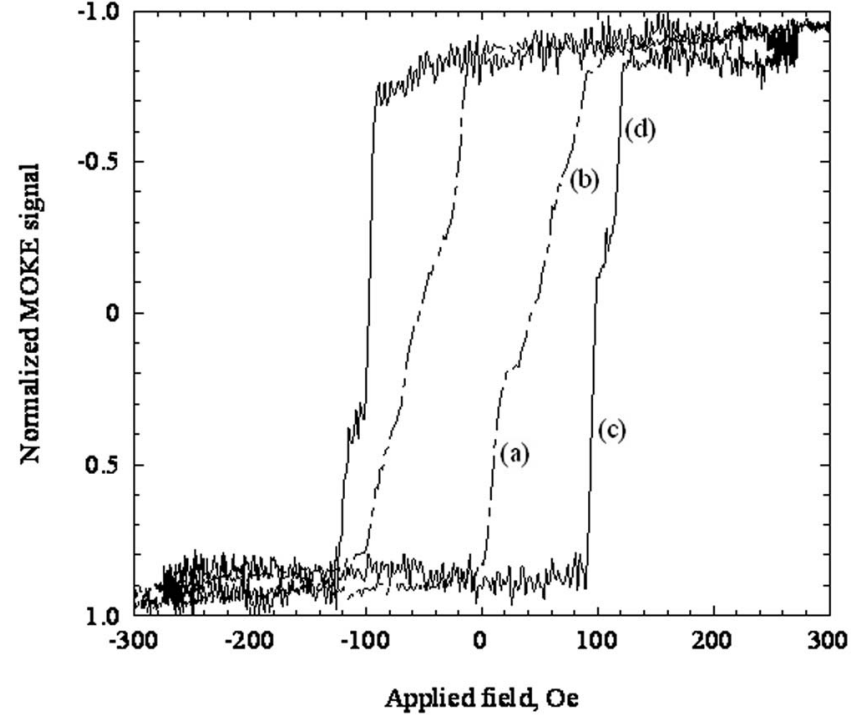

FIG. 6. MOKE hysteresis with the laser spot focused over the pad-wire junction (dashed line) and after the notch (solid line) for a $190 \mathrm{~nm}$ deep triangular notch in $385 \mathrm{~nm}$ wide nanowire.

ratio of notch depth to wire width. Furthermore, micromagnetic simulations suggest that at such notch depths, the domain wall is strongly pinned in the notch and, as the magnetic field increases, there is enough energy associated with this applied field to reverse the remainder of the wire without actually depinning the wall from the notch. While we have seen in Fig. 4 results to suggest that the precise geometry of the notch structure is relatively unimportant, Fig. 5 now suggests that increasing the notch depth does not necessarily yield a significantly greater pinning potential for the wall and can instead lead to further wall nucleation at the notch rather than the original wall simply depinning.

For asymmetric notch structures, the pinning behavior can also be seen to depend sensitively on the domain wall chirality. Figure 6 presents an example MOKE hysteresis loop from a $385 \mathrm{~nm}$ wire containing a $190 \mathrm{~nm}$ deep triangular notch structure, which suggests that there are two types of pinning interactions occurring at the notch. The MOKE loops are measured over many hundreds of field cycles and consequently represent the switching behavior averaged over hundreds of reversals. Figure 6 contains two hysteresis loops - the dashed line corresponds to depinning from the injection pad while the solid line corresponds to domain wall depinning from the notch. Considering head-to-head walls only, we can attribute the switching events at (a) and (b) to the pad switching at 12 Oe followed by domain wall injection into the nanowire occurring between 60 and $70 \mathrm{Oe}$, respectively. The switching events at (c) and (d) suggest two switching events measured with the MOKE laser spot located on the wire after the notch. The similarity in field values for the events (b) and (c) suggests that one type of event equates to a wall structure that is able to propagate along the full length of the wall and is relatively unaffected by the presence of a notch. Conversely, the switching event at (d) occurs at 130 Oe suggesting that another wall structure is pinned at this significantly higher magnetic field. We suggest this behavior is a direct consequence of how different wall 
(a)

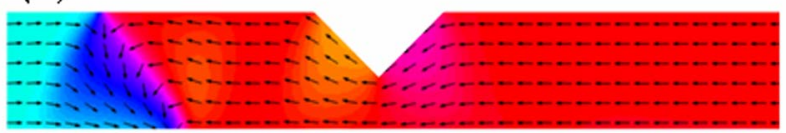

(b)

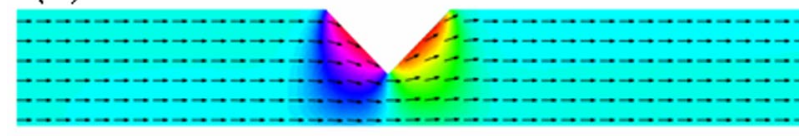

(c)

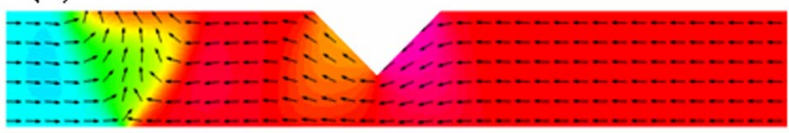

(d)

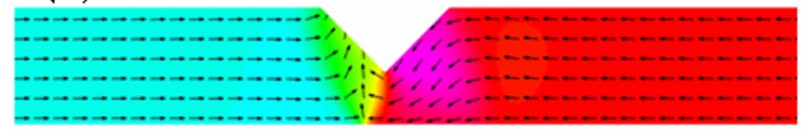

(e)

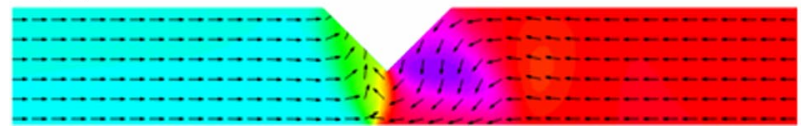

FIG. 7. (Color online) Quasistatic OOMMF micromagnetic simulations illustrating the chirality sensitive pinning behavior of transverse walls propagating in a $300 \mathrm{~nm}$ wire containing a $150 \mathrm{~nm}$ triangular notch. An initial field of $H_{x}=60$ Oe is applied since this corresponds to the average injection field of a $300 \mathrm{~nm}$ wire as determined from MOKE measurements. The down transverse wall (a) is not pinned by the notch at (b) 60 Oe and is able to propagate through the notch whereas the up wall (c) is significantly pinned by the notch at $60 \mathrm{Oe}$ (d) up to an applied field of $170 \mathrm{Oe}$ (e).

configurations interact with the spin structure around the notch. In a transverse wall, the spins are orientated at $90^{\circ}$ to the wire axis and there are two ways in which the magnetization can rotate within the wall since the spins can point either "up" or "down." These two wall structures are energetically degenerate and when injected from a symmetrical pad-wire interface both are equally probable. An asymmetric notch structure, however, will represent different pinning potentials depending on the chirality of the wall and will lift the energetic degeneracy of the wall structures. In Permalloy nanowires, the spins are largely constrained to lie along the long axis of the wire and to follow the local features of any patterned structures along the wire edge. If the spins in the domain wall lie in opposing directions to those spins around the edge of a notch then for energetic reasons one wall chirality may pin, while the opposite wall chirality may propagate directly through unpinned. ${ }^{14}$

We can see the dependence of domain wall configuration or chirality on pinning behavior in Fig. 7 , in which we present quasistatic OOMMF images for head-to-head walls interacting with a $150 \mathrm{~nm}$ deep triangular notch in a $300 \mathrm{~nm}$ wire. In this case, both walls are initially propagating at 60 Oe which corresponds to the injection field of a domain wall into a $300 \mathrm{~nm}$ wire and at this field the different pinning interactions of each wall structure are clear. First, the down transverse wall is not pinned by the notch and is able to propagate directly through, while the up transverse wall is pinned firmly at the entrance to the notch and remains significantly pinned until 170 Oe. The pinning behavior dependence upon wall chirality observed in Fig. 7 is in excellent agreement with the MOKE hysteresis loops presented in Fig. 6 , which were obtained from a triangular notch structure of a similar depth/wire ratio.

\section{CONCLUSION}

A combination of MOKE magnetometry and OOMMF micromagnetic simulations have allowed a systematic study into domain wall pinning behavior as a function of geometrical confinement within planar nanowires. The parameter space has been explored for two notch structure geometries and MOKE results suggest that depinning fields are relatively insensitive to the detailed geometry of the notch and instead the magnitude of depinning field of a wall from a notch structure is strongly influenced by the wire width. We also suggest that there is a limit to the increase in pinning potential of notches that is achieved by increasing the depth of the notch and that the wall chirality is significant in determining pinning behavior. These results are important in the optimization of field driven domain wall propagation and pinning behavior in nanowires.

${ }^{1}$ D. A. Allwood, G. Xiong, C. C. Faulkner, D. Atkinson, D. Petit, and R. P. Cowburn, Science 309, 1688 (2005).

${ }^{2}$ S. S. P. Parkin, U.S. Patent No.683 4005 (2004).

${ }^{3}$ M. Hayashi, L. Thomas, C. Rettner, R. Moriya, X. Jiang, and S. S. P. Parkin, Phys. Rev. Lett. 97, 207205 (2006).

${ }^{4}$ R. P. Cowburn, D. A. Allwood, G. Xiong, and M. D. Cooke, J. Appl. Phys. 91, 6949 (2002).

${ }^{5}$ K. J. Kirk, J. N. Chapman, S. McVitie, P. R. Aitchison, and C. D. W. Wilkinson, Appl. Phys. Lett. 75, 3683 (1999).

${ }^{6}$ D. A. Allwood, G. Xiong, M. D. Cooke, and R. P. Cowburn, J. Phys. D 36, 2175 (2003).

${ }^{7}$ http://math.nist.gov/oommf/

${ }^{8}$ To numerically calculate the domain wall equilibrium ground state energy per unit cross-sectional area following procedure was used. For a given wire width, a vortex domain wall was artificially introduced into a rectangular shaped element $10 \mathrm{~nm}$ thick and $6000 \mathrm{~nm}$ long and allowed to relax to its equilibrium configuration at zero applied field. A simulation was also performed with the wire at saturation, i.e., containing no domain wall. The energy of the domain wall was deduced by subtracting $E_{\text {total }}$ (wire containing domain wall) $-E_{\text {total }}$ (wire at saturation) to account for magnetostatic energy associated with the flat end shapes of the nanowire element. This value was then divided by the cross-sectional area to normalize all domain wall energies relative to one another.

${ }^{9}$ R. D. McMichael and M. J. Donahue, IEEE Trans. Magn. 33, 4167 (1997).

${ }^{10}$ Y. Nakatani, A. Thiaville, and J. Miltat, J. Magn. Magn. Mater. 290-291, 750 (2005).

${ }^{11}$ Y. Yokoyama, Y. Suzuki, S. Yuasa, K. Ando, K. Shigeto, T. Shinjo, P. Gogol, J. Miltat, A. Thiaville, T. Ono, and T. Kawagoe, J. Appl. Phys. 87, 5618 (2000).

${ }^{12}$ M. Klaui, H. Ehrke, U. Rudiger, T. Kasama, R. E. Dunin-Borkowski, D. Backes, L. J. Heyderman, C. A. F. Vaz, J. A. C. Bland, G. Faini, E. Cambril, and W. Wernsdorfer, Appl. Phys. Lett. 87, 102509 (2005).

${ }^{13}$ C. C. Faulkner, M. D. Cooke, D. A. Allwood, D. Petit, and D. Atkinson, J. Appl. Phys. 95, 6717 (2004).

${ }^{14}$ D. Atkinson, D. S. Eastwood, and L. K. Bogart, Appl. Phys. Lett. 92, 022510 (2008). 